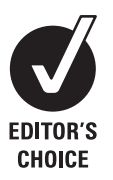

${ }^{1}$ Psychology Department, University of Guelph, Guelph, Ontario; ${ }^{2}$ Emergency Medicine, Kingston General Hospital, Kingston, Ontario

Correspondence to: Dr B A Morrongiello, Psychology Department, University of Guelph, Guelph, Ontario N1G 2W1, Canada; bmorrong@ uoguelph.ca

Accepted 3 February 2009

\title{
Identifying predictors of medically-attended injuries to young children: do child or parent behavioural attributes matter?
}

\author{
B A Morrongiello, ${ }^{1}$ M Corbett, ${ }^{1}$ R J Brison ${ }^{2}$
}

\begin{abstract}
Objective: To investigate whether one can differentiate injured and uninjured young children based on child behavioural attributes or indices of caregiver supervision.

Method: A matched case-control design was used in which case participants were children presenting to an emergency department for treatment for an injury and age/sex matched control participants presented for illness-related reasons. During structured phone interviews about supervision parents reported on general supervisory practices (standardised questionnaire) and specific practices corresponding to time of injury (cases) or the last time their child engaged in the activity that incited their match's injury (controls). Parents also reported on child behavioural attributes that have been linked to child risk taking in prior research (inhibitory control, sensation seeking).
\end{abstract}

Results: Results revealed no group differences in child behavioural attributes; however, the control group received more supervision both in general $(\mathrm{OR}=4.82$, $95 \% \mathrm{Cl} 1.89$ to 12.33 ) and during the specified activity that led to injury in cases $(\mathrm{OR}=5.38,95 \% \mathrm{Cl} 2.13$ to 13.58).

Conclusion: These findings confirm past speculation that caregiver supervision influences children's risk of medically-attended injury and highlight the importance of targeting supervision in child-injury prevention interventions.

Unintentional injury is a pressing health issue for children. In many developed nations it is the leading cause of death for children over the age of 1 year $^{12}$ and significantly contributes to hospitalisation. ${ }^{3}$ The economic costs are staggering (eg, \$5.7 billion was spent in 2000 treating the injuries of American children under the age of $10^{4}$ ). The personal costs are immeasurable.

For young children, because many injuries occur in the home when they are supposedly being monitored by a caregiver, ${ }^{5}{ }^{6}$ studies to identify injury-risk factors have considered both child behavioural attributes and caregiver safety practices. Two child behavioural characteristics that influence injury risk are sensation seeking (ie, preference for novel and emotionally arousing risk activities) and inhibitory control (ie, capacity to follow rules and resist doing something one wants to). Children high in sensation seeking typically show increased risk taking, ${ }^{7-10}$ whereas those high in inhibitory control show decreased risk taking. ${ }^{11}$ No studies have examined whether these behavioural attributes have sufficient predictive power to differentiate injured from uninjured children; this issue was addressed in the present study. Based on prior research, we hypothesised that children low in inhibitory control or high in sensation seeking would be more likely to be injured than those not possessing these attributes.

Recent studies of caregiver safety practices have focused on supervision, and sought to determine what constitutes "adequate" supervision to ensure a child's safety and how quality of supervision affects children's injury risk. ${ }^{13}$ A prospective study of young children's in-home minor injuries indicates that quality of supervision affects frequency of injury. ${ }^{7}$ The present study sought to extend these findings by testing the hypothesis that one can differentiate children with medicallyattended injuries from uninjured children based on caregiver reports about supervision. A matched case-control design was used, with parents of case (injured) and control (uninjured) children reporting on child behavioural attributes and supervision. Including both child- and parent-based risk factors in the same study provided a unique opportunity to examine the relative role of each in predicting child-injury status.

\section{METHODS \\ Design}

A case-control design was used, including injured and uninjured children, respectively. Cases and controls were matched for sex and age (within 6 months) because these variables have been associated with differences in supervision and injury. ${ }^{2} 71516$

\section{Participants}

The sample consisted of 35 pairs of children $(\mathrm{n}=70)$ including 11 female and 24 male pairs; this sex difference is representative of the typical finding that males are more frequently injured than females. ${ }^{12}$ Recruitment occurred at the Emergency Department (ED) at Kingston General Hospital in Kingston, Ontario, Canada during 2006 and through the summer of 2007 . No attempt was made to recruit all possible injured children who met the criteria. Rather, the availability of the research assistant determined those children who were recruited. This ED participates in the Canadian Hospitals Injury Reporting and Prevention Program (CHIRPP) network that tracks child injuries and solicits parental consent for contact for research purposes. Past findings regarding rankings of injury patterns and mechanisms, objects, locations, and activities that relate to child injury at this CHIRPP centre have been shown to 
be representative of those obtained with a national Canadian sample. ${ }^{17}$ The recruitment criteria included: (1) normally developing (confirmed by parental report) boys and girls between the ages of 2 and 6.5 years who had English-speaking parents; (2) not seeking treatment for a life-threatening injury or illness (as judged by the triage nurse); and (3) injuries were judged by the attending physician to not be intentional ones. The mean ages of the cases (injured) and control (uninjured) groups were 43.97 months $(S D=14.14)$ and 43.88 months $(\mathrm{SD}=14.13)$, respectively; table 1 lists household income and highest level of parental education for each group.

\section{Measures}

In addition to demographic questions about family income and parental education, the following measures were completed during a telephone interview.

\section{Supervision at time of injury interview}

This structured interview, delivered only to parents of the injured children (cases), was used to establish the level of supervision at the time of the injury. Parents began by telling about the type of injury (eg, tripped on carpet and banged head on the wall as child fell; rolled off couch and hit hand on a toy and cut it; turned on the hot water tap and burned hand) and then answered questions about supervision. A single dichotomous item was used to establish whether the child was being supervised at the time. If applicable, a follow-up open-ended item determined who was supervising. Following this, three items were presented to determine the level of supervision at the time of the injury with regard to watching, listening, and proximity, as outlined in the supervision taxonomy developed by Morrongiello et al. ${ }^{8}$ Items for watching and listening presented three alternatives (available from the first author on request) indicating the extent of the supervisory behaviour, with scores ranging from 1 to 3 (eg, watching: $3=$ the supervisor had the child in view and was watching him/her at the time of injury); the physical contact item presented four alternatives (eg, 4 = supervisor was in physical contact with the child at the time of injury). The scores for these three items were summed to form the "overall supervision" score (0-10), with higher scores indicating closer supervision in terms of watching, listening, and proximity.

Table 1 Percent of case $(n=35)$ and control $(n=35)$ samples in each education and income level

\begin{tabular}{lcl}
\hline & \% Cases $(\mathbf{n})$ & \% Controls (n) \\
\hline Father's education & & \\
$\quad$ Some or completed high school & $29.4(10)$ & $17.2(5)$ \\
$\quad$ Some or completed college/university & $64.7(22)$ & $65.5(19)$ \\
$\quad$ Some or completed graduate degree or & $5.9(2)$ & $17.2(5)$ \\
$\quad$ postgraduate training & & \\
Mother's education & $26.5(9)$ & $22.9(8)$ \\
$\quad$ Some or completed high school & $70.6(24)$ & $68.6(24)$ \\
Some or completed college/university & $2.9(1)$ & $8.6(3)$ \\
Some or completed graduate degree or & & \\
postgraduate training & & \\
Household income (\$) & $3.2(1)$ & $18.2(6)$ \\
$\quad$ Below 20 000 & $29.0(9)$ & $21.2(7)$ \\
$20000-39999$ & $25.8(8)$ & $18.2(6)$ \\
$40000-59000$ & $9.7(3)$ & $21.2(7)$ \\
$60000-79999$ & $32.3(10)$ & $21.2(7)$ \\
Above 80000 &
\end{tabular}

Supervision at time of activity interview

This structured interview was administered solely to parents of the uninjured children (controls); it paralleled that for the case children with the following exceptions. The research assistant reviewed the interview response of the control child's matched case counterpart in order to determine the activity that incited the injury. Parents of control children were then asked to consider the last time that their own child engaged in this specific activity, to indicate when this was, and to report on supervision at that time.

Parent Supervision Attributes Profile Questionnaire-Home (PSAPQ) The PSAPQ is a two-part scale that shows both strong reliability and validity as a measure of parental supervisory practices at home that are relevant to young children's injury risk. ${ }^{18}{ }^{19}$ Part I deals with parental attitudes towards closely supervising. Parents are asked to rate the extent to which they agree or disagree with each of 20 items on a 5-point Likert scale $(1=$ strongly disagree, $5=$ strongly agree). Subscale scores are obtained for "protectiveness of child" (internal reliability: $\alpha=0.72$ for this sample), "vigilance about hazards" ( $\alpha=0.68)$, and "worry about child injury" $(\alpha=0.68)$ by averaging the scores of applicable items; higher scores indicate higher levels of the attitude under consideration. An overall score is generated by averaging scores of all items $(\alpha=0.72)$. Part II contains 14 items, each stating an activity that a child would typically engage in at home (eg, eating breakfast, taking a bath). Responses given on a 5-point Likert scale indicate the level of supervision typically provided ( $1=$ "I'm typically in another room and I go to my child when he/she calls for me", $5=$ "I'm typically in the same room and within arm's reach of my child"). Higher scores indicate higher levels of supervision. These items comprise three subscales: "play activities" (eg, playing in his/her bedroom, $\alpha=0.81$ for this sample), "self-care activities" (eg, brushing his/her teeth, $\alpha=0.77$ ), and "risk activities" (eg, climbing on furniture, $\alpha=0.70$ ). Subscale scores are determined by averaging applicable items, after any non-applicable items are excluded; in this sample, the risk activities subscale had to be eliminated from analysis due to many parents identifying these as non-applicable items.

\section{Sensation Seeking Scale for Children-Toddlers (SSSC)}

The SSSC served as an index of sensation seeking. ${ }^{20}$ For each of 24 items, respondents are asked to select the one of two alternatives that better represents what their child would prefer to do. Items are designed such that one alternative presents a daring or arousing behaviour $(\mathrm{eg}$, ride a tricycle fast, score $=1$ ) and the other presents a more reserved behaviour (eg, ride a tricycle slowly, score $=0$ ). Summing across items yields an overall score, with higher scores indicating greater sensation seeking. Acceptable internal reliability was demonstrated in this sample $(\alpha=0.72)$.

\section{Early Childhood Behaviour Questionnaire (ECBO)}

The inhibitory control subscale of the $\mathrm{ECBO}^{21}$ was used. For each of 12 items, parents rate on a 7-point Likert scale $(1=$ never, $7=$ always $)$ how frequently their child engages in the behaviour (eg, "When asked to do so, how often is your child able to stop an ongoing activity?"). Summing across items yields a total score, with higher scores indicating greater inhibitory control ( $\alpha=0.87$ in this sample). 


\section{Procedure}

Once a case was identified, a parent of the injured child was called and a time for a telephone interview was established; calls occurred within 2 weeks of the ED visit. Recent ED records (within a month of the ED visit date) were then searched for a matched control child who was of the same sex, within 6 months of age, and had recently presented for a non-injury related reason (eg, fever, stomach ache). When a match was found, his/her parent was contacted and a telephone interview was scheduled. The interview was conducted with whichever parent was supervising at the time of injury for cases and with the corresponding parent for controls; for the two cases for which the supervisor at the time of injury was not a parent, the parent to be interviewed was randomly determined.

During the telephone call, which lasted approximately 30 minutes, the interview and questionnaires were delivered in random order. Due to the nature of the interviews, the research assistant was not blind to the condition of the parent's child. However, biases were obviated by the structured format of the interviews and questionnaires. Prior to their participation, parents of all children granted verbal consent; all procedures and materials were approved by the Research Ethics Boards of the University of Guelph and Queen's University.

\section{Data analysis approach}

Descriptive and parametric statistics were applied to the data, with the general aim being to compare scores obtained by cases and controls to test our hypotheses and identify significant group differences in child behavioural attributes and aspects of parent supervision.

\section{RESULTS}

Bivariate analyses did not reveal any significant differences between the groups $(p>0.19)$ for any of the demographic characteristics listed in table 1; these were not therefore included as covariates in any analyses. Subsequent analyses focused on testing for hypothesised group differences in child behavioural attributes and parent supervision. As noted in prior research, ${ }^{22}$ when using a matched case-control methodology it is important to account for the matching process by assessing for any interaction between matching variables and other predictors to predict outcome measures. If such effects are evidenced then they need to be treated as covariates in subsequent analyses. To this end we conducted a series of logistic regression analyses assessing for any interactions between each predictor (in separate regressions) with both age and sex variables, predicting group membership. Results indicated that matching variables did not interact with other predictors; accounting for our matched design in subsequent analyses was therefore unnecessary.

\section{Child behavioural attributes}

To discern whether differences in the child behavioural attributes of sensation seeking and inhibitory control existed between the injured and control groups, sensation seeking and inhibitory control scores were compared using one-way between-groups analyses of variance (ANOVA). Case and control children did not significantly differ in sensation seeking $($ mean $(\mathrm{M})=12.51$ and $12.25, \mathrm{SD}=4.24$ and 4.31 , respectively, $\left.\mathrm{F}(1,68)=0.07, \quad \mathrm{p}=0.80, \quad \eta_{\mathrm{p}}{ }^{2}=0.00\right)$ or inhibitory control $(\mathrm{M}=4.73$ and $4.74, \mathrm{SD}=0.84$ and 1.11 , respectively, $\left.\mathrm{F}(1,68)=0.00, \mathrm{p}=0.96, \eta_{\mathrm{p}}{ }^{2}=0.00\right)$. Thus, there were no group differences in child behavioural attributes.

\section{Parent supervision}

\section{Attitudes about supervision}

An ANOVA with group as a between-subjects factor was applied to the PSAPQ Part I subscale scores, which reflect parental attitudes concerning supervision. As table 2 shows, parents of the control group scored significantly higher than parents of the injured group on all subscales of the PSAPQ Part $\mathrm{I}$ : protectiveness, $\mathrm{F}(1,68)=35.56, \mathrm{p}=0.001, \eta_{\mathrm{p}}{ }^{2}=0.34$; vigilance, $F(1,68)=22.50, \quad p=0.001, \quad \eta_{p}^{2}=0.25 ;$ and worry, $F(1,68)=16.97, p=0.001, \eta_{p}{ }^{2}=0.20$. Furthermore, parents of the control groups scored significantly higher overall on Part I, $\mathrm{F}(1,68)=31.56, \mathrm{p}=0.001, \eta_{\mathrm{p}}{ }^{2}=0.32$.

\section{Typical level of supervision}

In order to determine whether differences existed between the groups in the degree of supervision typically provided, scores on Part II of the PSAPQ were examined with one-way betweengroups ANOVAs (see table 2). The level of supervision parents provided for uninjured children significantly exceeded that reported by parents of injured children for both the play activities subscale $\left(F(1,68)=16.91, p=0.000, \eta_{p}{ }^{2}=0.20\right)$ and self-care activities subscale $\left(F(1,68)=12.28, p=0.001, \eta_{p}{ }^{2}=0.15\right)$.

\section{Supervision at time of injury or corresponding activity}

To determine whether the average recall interval (ie, time since the child engaged in the activity until the parent completed the interview) for the control parents was within the two-week period used for case parents, control parents were asked how recently their child engaged in the behaviour inciting their match's injury. Results revealed that $100 \%$ of the control children had engaged in this behaviour recently $(M=9.44$ days, $\mathrm{SD}=8.08)$. Thus, any group difference in supervision is not likely to reflect differences in recall intervals across groups.

Parents were asked to report if a supervisor was present at the time of injury (case) or last activity (control). For cases, $18 \%$ of the children were not being supervised at all (ie, child was presumed to know how to behave and to not need to be watched or checked on) at the time of injury. In contrast none of the control parents reported that their child was not directly supervised the last time the child engaged in the activity of interest, $\chi^{2}(1, n=64)=5.84, p=0.016$. For those who reported supervisors were present, there was no significant difference between case and control parents in who was supervising at the time of injury or last activity, $\chi^{2}(2, \mathrm{n}=65)=3.47, \mathrm{p}>0.25$, with virtually all supervisors being a parent $(96 \%$ and $99 \%$ for cases and controls, respectively).

Separate one-way between-group ANOVAs were conducted to compare the supervision scores of case and control parents for the dimensions of watching, listening and proximity, as well as overall score. As table 2 shows, there were significant differences between groups for watching, $F(1,68)=12.86, p=0.001$, $\eta_{p}{ }^{2}=0.31 ;$ listening, $\quad F(1,68)=30.75, \quad p=0.001, \quad \eta_{p}{ }^{2}=0.31 ;$ proximity, $\mathrm{F}(1,68)=21.03, \mathrm{p}=0.001, \eta_{\mathrm{p}}{ }^{2}=0.24$; and overall supervision, $F(1,68)=38.57, p=0.001, \eta_{p}{ }^{2}=0.36$, with the control group scoring higher than the case group for all measures of supervision.

\section{Predictors of injury status}

To identify factors that predicted having a medically-attended injury (case group), a logistic regression was conducted. PSAPQ Part I scores were included to assess for the influence of attitudes about supervision on injury status. This measure is important because it assesses parental influences that cannot be 
Table 2 Mean scores of child attributes, Parent Supervision Attributes Profile Questionnaire-Home (PSAPQ) (Parts I and II), and reported supervision behaviours (watching, listening, proximity and overall score) at the time of injury (case) or last activity (control)

\begin{tabular}{|c|c|c|c|c|c|c|}
\hline Measure & Subscale/behaviour & $\begin{array}{l}\text { Cases } \\
(\mathrm{n}=35)\end{array}$ & $\begin{array}{l}\text { Controls } \\
(n=35)\end{array}$ & $\mathbf{F}$ & $\eta_{p}^{2}$ & p Value \\
\hline \multirow[t]{2}{*}{ Child attribute } & Sensation seeking & 12.51 & 12.25 & 0.07 & 0.00 & 0.795 \\
\hline & Inhibitory control & 4.73 & 4.74 & 0.00 & 0.00 & 0.963 \\
\hline \multirow[t]{4}{*}{ PSAPO Part I } & Protectiveness & 3.63 & 4.15 & 35.56 & 0.34 & 0.001 \\
\hline & Vigilance & 3.25 & 3.83 & 22.50 & 0.25 & 0.001 \\
\hline & Worry & 2.50 & 3.27 & 16.97 & 0.20 & 0.001 \\
\hline & Overall & 3.56 & 3.88 & 31.56 & 0.32 & 0.001 \\
\hline \multirow[t]{2}{*}{ PSAPQ Part II } & Play activities & 3.11 & 3.69 & 16.91 & 0.20 & 0.001 \\
\hline & Self-care activities & 3.79 & 4.29 & 12.28 & 0.15 & 0.001 \\
\hline \multirow[t]{4}{*}{ Supervision } & Watching & 1.83 & 2.69 & 30.66 & 0.31 & 0.001 \\
\hline & Listening & 2.14 & 2.91 & 30.75 & 0.31 & 0.001 \\
\hline & Proximity & 1.63 & 2.46 & 21.03 & 0.24 & 0.001 \\
\hline & Overall score & 5.60 & 8.06 & 38.57 & 0.36 & 0.001 \\
\hline
\end{tabular}

captured by behavioural measures but have proven important in predicting minor injury outcomes. ${ }^{18}$ Part II scores were not included in the model because, although these are good proxy measures for typical supervisory activities, in the presence of direct reports of supervisory behaviours at the time of the injury-risk activity, these scores were considered redundant. The initial analysis plan was to include individual PSAPQ subscale scores and individual supervisory scores, however regressions diagnostics indicated that a regression model including all of these predictors would be influenced by multicollinearity. PSAPQ scores were correlated with each other as were the supervision at time of injury scores; including them all in the same model produced large inflations in standard errors of parameter estimates. ${ }^{23}$ Therefore, as suggested in past research, ${ }^{24}$ we chose to use aggregate scores rather than individual subscale scores to reduce the influence of multicollinearity. Regression diagnostics indicated that this approach was effective at reducing multicollinearity (eg, aggregate scores were not highly correlated and standard errors were not inflated). Demographic variables (parental education and income) were also assessed for their influence on injury status during the model building process. Although the aforementioned $\chi^{2}$ analyses did not reveal any main effects of these factors, we also wished to assess for any interaction effects with supervision variables. A series of logistic regression analyses were conducted to determine if we could predict injury status from centred supervision variables, demographic variables, or their product terms entered in one block. Results did not reveal any significant interactions. Thus, demographic variables were not included in the final model. In order that a one-unit change in all predictors could be meaningfully interpreted, PSAPQ Part I scores (attitude towards closely supervising) and overall actual supervision scores were transformed into zscores prior to analysis. PSAPQ Part I overall score, and overall actual supervision scores for the time of injury or activity were entered as predictors. Results (see table 3 ) showed that the full model was statistically significant $\left(\chi^{2}(3, n=70)=46.27\right.$, $p=0.001)$, indicating that the predictors reliably distinguished between case and control groups. According to the Wald criterion, PSAPQ Part I overall score (Wald $(1, \mathrm{n}=70)=10.80$, $\mathrm{p}=0.001)$ and overall actual supervision scores at time of injury or activity (Wald $(1, \mathrm{n}=70)=12.72, \mathrm{p}=0.001)$, significantly predicted group assignment. Odds ratios showed that as values on PSAPQ Part I (positive attitudes towards closely supervising) increased by 1 standard deviation, the odds of being in the control group were 4.82 times higher. Additionally, as overall supervision increased by 1 standard deviation, the odds of being in the control group were 5.38 times higher. Thus, both parents' supervision attitudes and practices each significantly differentiated injured from uninjured children.

\section{DISCUSSION}

The present study used a matched case-control design to explore how child behavioural attributes (sensation seeking and inhibitory control) and parent supervision (attitudes, practices) influence children's risk of experiencing a medically-attended injury. There is a long history of research exploring relations between child attributes and injury risk, with a primary focus on studying aspects of temperament and personality. ${ }^{25} 26$ Although past studies have related sensation seeking and inhibitory control to children's risk taking, the current findings suggest that these attributes do not in and of themselves differentiate children who are at risk for injury, at least not at these young ages. Similar conclusions have been noted for impulsivity, namely that the evidence linking impulsivity to injury risk for younger age children is less consistent than the findings obtained with older children. ${ }^{7}$ It may be that the impact of child attributes on injury risk increases in scope and consistency across developmental stages as supervision naturally declines and children are increasingly left to decide about risk activities independently of direct adult input. Injuries during the elementary-school years, for example, are more likely to occur when children are unsupervised and on their own or with peers. ${ }^{6}{ }^{27}$ At younger ages, the mere presence in the home of a parent, even one not directly supervising, often deters risk behaviours, ${ }^{8}$ and direct supervision can significantly reduce the risk of injury to children who have temperamental attributes that increase their injury risk. ${ }^{28}{ }^{29}$ Hence, because young children are often in the vicinity of potential supervisors, this may reduce the relative impact child behavioural attributes have on their risk of injury. With increasing age there are changes in the expression of temperament and increasing independence from supervisors, which may result in behavioural attributes having a more direct influence on children's risk of injury. Hence, 
Table 3 Logistic regression results $(n=70)$

\begin{tabular}{llllll}
\hline Measures & $\boldsymbol{B}$ & $\mathbf{S E}$ & Wald & $\mathbf{p}$ Value & Odds Ratio (95\% CI) \\
\hline PSAPQ overall & 1.573 & 0.479 & 10.796 & 0.001 & $4.823(1.887$ to 12.330) \\
Overall supervision & 1.683 & 0.472 & 12.717 & 0.001 & 5.384 (2.134 to 13.581) \\
\hline
\end{tabular}

although child behavioural attributes did not predict medically attended injury in this study, significant effects might be obtained for children at older ages.

Interestingly, significant differences between parents of injured and uninjured children emerged both for attitudes towards closely supervising and reported supervision practices. Specifically, parents of injured children scored lower on a measure of positive attitudes towards closely supervising (PSAPQ) and, consistent with this, they reported lower levels of supervisory behaviours (watching, listening, proximity to child). Although a previous study showed systematic relations between in-home supervision and children's frequency of minor injuries that parents treated at home, ${ }^{78}$ to our knowledge this is the first study to link parent supervision and likelihood of a young child experiencing an injury that results in seeking medical treatment. Lower levels of supervision resulted in a fivefold increase in risk of medicallyattended injury $(\mathrm{OR}=5.39)$ for young children.

From an intervention perspective, the demonstration that supervision functions as a protective factor with respect to child injury is significant because it confirms the importance of developing interventions that target parent supervision. In the area of child-injury prevention, considerable research and intervention efforts have been expended to identify ways to promote parents' hazard-reduction practices in the home (eg, use of safety devices, removal of hazards), but comparatively little is known about how to promote closer supervision practices. ${ }^{30}{ }^{31}$ Certainly, environmental modifications to eliminate access to hazards in the home environment can effectively reduce young children's risk of injury. However, there is no substitute for supervision, particularly given that not all hazards can be removed and devices that serve to reduce access to hazards at some ages (eg, stair gates) can actually become hazards and increase risk of injury as the child achieves subsequent developmental milestones (eg, climbing on stair gates) or if there are older siblings in the home who already have achieved these milestones. Moreover, it may even be that encouraging hazardreduction practices results in decreased supervision, because parents develop a false sense of security about their child's safety and demonstrate risk compensation in reaction to these safety devices or practices. ${ }^{32}$ For a variety of reasons, therefore, it is essential that child-injury prevention efforts be expanded to focus on identifying ways to promote closer supervision of young children by parents. Importantly, recent successes in doing so confirm the feasibility of this prevention approach. ${ }^{33} 34$

\section{Limitations}

Several limitations merit consideration in interpreting the findings and planning future studies. First, the data are based on self-reports. Although past research shows that parents are not hesitant to report about times when they are not supervising, ${ }^{8}$ and that questionnaire-based reports about supervision mirror parents' supervisory practices, ${ }^{18} 19$ one cannot be certain that the parents in this study accurately reported on supervision behaviours. Second, the findings are based on a small suburban sample that may be unique in some ways. Although past research at this hospital shows child injury patterns that are representative of what is found nationally, ${ }^{17}$ this does not confirm that the current findings are generalisable to the

\section{What is already known on this topic}

- Child behavioural attributes and parent supervision can influence young children's risk of minor home injuries.

- A case-control study examining the predictive value of child behavioural attributes and parent supervision for differentiating injured from uninjured young children has not been previously conducted but is a logical next step.

\section{What this study adds}

- For young children, behavioural attributes did not predict injury group status, but indices of parents' attitudes towards closely supervising and supervisory practices each differentiated injured from uninjured groups.

- Child behavioural attributes may gain in importance as children age and are less directly supervised by parents.

- Expanding injury-prevention initiatives to target parent supervision practices may prove effective to reduce injuries to young children.

broader population of parents. Finally, this was a sample of convenience. Hence, it is possible that there is some selection bias operating that we are not aware of and that may contribute to explain the group differences. For example, parents who take their child to the ED for treatment for illness may be more likely to supervise than parents who are less likely to visit the ED for child illness. In this case, different results might have been obtained if community-recruited controls, as opposed to hospital-recruited controls, had been included. Thus, while the present results provide evidence of the protective role supervision can play in preventing childhood injury, it would be useful to extend this study by using a larger more demographically diverse population, including a community-based control group, and incorporating observational measures of supervision to confirm self-reports.

In conclusion, the present findings add to the accumulating evidence linking parent supervision to young children's risk of injury and highlight the importance of developing intervention strategies that succeed in motivating parents to closely supervise young children. The fact that child behavioural attributes did not differentiate injured from uninjured children may relate to the young ages of the children in this study; these factors may gain in importance across developmental stages as direct supervision naturally declines in service of promoting children's independence and autonomy.

Acknowledgements: The authors extend their thanks to Deb Emmerton and Amy Pickett for assistance with data collection and to the parents for their participation.

Funding: This research was supported by grants from the Social Sciences and Humanities Research Council of Canada.

Competing interests: None.

Ethics approval: Ethics approval was obtained. 


\section{REFERENCES}

1. Canadian Institute of Child Health. The health of Canada's children, 3rd edn. Ottawa: Canadian Institute of Child Health, 2002.

2. National Center for Injury Prevention and Control. WISOARS (web-based injury statistics query and reporting system). http://webappa.cdc.gov/sasweb/ncipc/ leadcaus10.html (accessed 18 June 2009).

3. Scheidt P, Harel Y, Tumble A, et al. The epidemiology of nonfatal injuries among U.S. children and youth. Am J Public Health 1994;85:932-8

4. CDC. Medical expenditures attributable to injury, United States. MMWR Morb Mortal Wkly Rep 2000;53:1-4

5. Rivara FP. Developmental and behavioral issues in childhood injury prevention. J Dev Behav Pediatr 1995;16:362-70.

6. Shanon A, Bashaw B, Lewis J, et al. Nonfatal childhood injuries: a survey at the Children's Hospital of Eastern Ontario. Med Assoc J 1992;146:361-5.

7. Morrongiello BA, Ondejko L, Littlejohn A. Understanding toddlers' in-home injuries: I. Context, correlates, and determinants. J Pediatr Psychol 2004;29:415-31.

8. Morrongiello BA, Ondejko L, Littlejohn A. Understanding toddlers' in-home injuries: II. Examining parental strategies and their efficacy for managing child injury risk. J Pediatr Psychol 2004;29:433-46.

9. Morrongiello BA, Sedore L. The influence of child attributes and social-situational context on school-age children's risk taking behaviors that can lead to injury. $J$ App/ Dev Psychol 2005:26:347-61.

10. Morrongiello BA, Walpole B, Lasenby J. Understanding children's injury-risk behavior: wearing safety gear can lead to increased risk taking. Accid Anal Prev 2007:39:618-23.

11. Plumert JM, Schwebel DC. Social and temperamental influences on children's overestimation of their physical abilities: link to accidental injuries. J Exp Child Psychol 1997:67:317-37.

12. Schwebel DC, Plumert JM. Longitudinal and concurrent relations among temperament, ability estimation and injury proneness. Child Dev 1999;70:700-12.

13. Gitanjali S, Morrongiello BA, Haynie D, et al. The role of supervision in child injury risk: definition, conceptual, and measurement issues. Inj Control Saf Promot 2004:11:17-22.

14. Morrongiello BA. Caregiver supervision and child-injury risk: I. Issues in defining and measuring supervision; II. Findings and directions for future research. J Pediatr Psychol 2005;30:536-52.

15. Dal Santo JA, Goodman RM, Glik D, et al. Childhood unintentional injuries: factors predicting injury risk among preschoolers. J Pediatr Psychol 2004;29:273-83.

16. Peterson L, Ewigman B, Kivlahan C. Judgments regarding appropriate child supervision to prevent injury: the role of environmental risk and child age. Child Dev 1993;64:934-50.

17. Pickett W, Brison RJ, Mackenzie SG, et al. Youth injury in the Canadian Hospitals Injury Reporting and Prevention Program: do they represent the Canadian experience? Inj Prev 2000;6:9-15.
18. Morrongiello BA, Corbett M. The Parent Supervision Attributes Profile Questionnaire: a measure of supervision relevant to children's risk of unintentional injury. Inj Prev 2006;12:19-23.

19. Morrongiello BA, House K. Measuring parental attributes and supervision behaviors relevant to child injury risk: examining the usefulness of questionnaire measures. Inj Prev 2004;10:114-8.

20. Morrongiello BA, Lasenby J. Finding the daredevils: development of a sensation seeking scale for children that is relevant to physical risk taking. Accid Anal Prev 2006;38:1101-6.

21. Putnam SP, Gartstein MA, Rothbart MK. Measurement of fine-grained aspects of toddler temperament: the Early Childhood Behavior Questionnaire. Infant Behav Dev 2006;29:386-401.

22. Thompson SG. Modelling in matched case-control studies in epidemiology. The Statistician 1986;35:237-44.

23. Pedhazur EJ. Multiple regression in behavioral research: explanation and prediction. Orlando: Harcourt Brace College Publishers, 1997.

24. Tabachnick BG, Fidell, LS. Using multivariate statistics, 3rd edn. New York: HarperCollins College Publishers, 1996

25. Morrongiello BA, Lasenby-Lessard J. Psychological determinants of risk taking by children: an integrative model and implications for interventions. Inj Prev 2007;13:20-5.

26. Schwebel D, Gaines J. Pediatric unintentional injury: behavioral risk factors and implications for prevention. J Dev Behav Pediatr 2007;28:245-54.

27. Morrongiello BA. Preventing unintentional injuries among children. In: Determinants of health in Canada. Ottawa: Multimondes Editions, 1997:37-74.

28. Morrongiello BA, Klemencic N, Corbett M. Interactions between child behavior patterns and parent supervision: implications for children's risk of unintentional injury. Child Dev 2008:79:627-38.

29. Schwebel DC, Bounds ML. The role of parents and temperament on children's estimation of physical ability: links to unintentional injury prevention. J Pediatr Psychol 2003:28:505-16.

30. Brown K, Roberts M, Boles R. Effects of parental viewing of children's risk behavior on home safety practices. J Pediatr Psychol 2005;30:571-80.

31. Morrongiello BA, Lasenby J. Supervision as a behavioral approach to reducing injury risk. In: Gielen A, Sleet D, DiClemente R, eds. Handbook of injury prevention behavior change theories, methods, and applications. New York: Jossey-Bass, 2005:395-418.

32. Morrongiello BA, Major K. Influence of safety gear on parental perceptions of injury risk and tolerance or children's risk taking. Inj Prev 2002;8:27-31.

33. Brown K, Roberts M, Boles R. Effects of parental viewing of children's risk behavior on home safety practices. J Pediatr Psychol 2005;30:571-80.

34. Morrongiello BA, Zdzieborski D, Sandomierski M, et al. Video messaging: what works to persuade mothers to supervise young children more closely to reduce injuries? Soc Sci Med 2009;68:1030-7. 